\title{
Transurethral resection of bladder tumour (TURBT)
}

\author{
Lawrence H. C. Kim ${ }^{1,2}$, Manish I. Patel ${ }^{1,2}$ \\ ${ }^{1}$ Department of Urology, Westmead Hospital, Sydney, Australia; ${ }^{2}$ Discipline of Surgery, Sydney Medical School, University of Sydney, Sydney, \\ Australia \\ Contributions: (I) Conception and design: All authors; (II) Administrative support: None; (III) Provision of study material or patients: All authors; \\ (IV) Collection and assembly of data: LHC Kim; (V) Data analysis and interpretation: All authors; (VI) Manuscript writing: All authors; (VII) Final \\ approval of manuscript: All authors. \\ Correspondence to: Professor Manish I. Patel. Suite 17, Westmead Private Hospital, Westmead NSW 2145, Australia. Email: mpatel@med.usyd.edu.au.
}

\begin{abstract}
The goals of transurethral resection of bladder tumour (TURBT) are to identify and eradicate visualized bladder tumour if technically safe and feasible and to obtain a specimen of satisfactory quality to enable accurate histological diagnosis. In the setting of high grade bladder tumour this generally entails the inclusion of detrusor muscle and assessment for the presence of associated carcinoma in situ (CIS), lymphovascular involvement or any variant form of bladder cancer. This will assist in determining risk stratification and prognostication of the bladder cancer and guides further treatment planning. Conversely, if suboptimal TURBT is performed there will be detrimental consequences on patient outcomes in regards to undergrading or understaging, increased recurrence or progression, and subsequently need for further treatments including more invasive interventions. This review article firstly summarises the key principles and complications of TURBT, as well as significance of re-TURBT. We also discuss a number of modifications and advances in detection technology and resection techniques that have shown to improve perioperative as well as pathological and oncological outcomes of bladder cancer. They include enhanced cystoscopy such as blue light cystoscopy (BLC), narrow band imaging (NBI) and en bloc resection of bladder tumour (ERBT) technique using various types of energy source.
\end{abstract}

Keywords: Transurethral resection of bladder tumour (TURBT); non-muscle invasive bladder cancer (NMIBC); carcinoma in situ (CIS); blue light cystoscopy (BLC); narrow beam imaging (NBI); en bloc resection of bladder tumour technique (ERBT technique)

Submitted Jul 25, 2019. Accepted for publication Sep 17, 2019.

doi: $10.21037 /$ tau.2019.09.38

View this article at: http://dx.doi.org/10.21037/tau.2019.09.38

\section{Introduction}

Bladder cancer is the fourth most common malignancy and eighth most common cancer risk deaths among men in the USA (1). Approximately $75 \%$ of patients present with nonmuscle invasive bladder cancer (NMIBC) in which cancer involves only the urothelium or lamina propria without invasion to the detrusor muscle (2). It is also known to be the very costly cancer to treat due to high recurrence rate and its protracted course (3). One of the most likely explanations for this is thought to be due to suboptimal quality of transurethral resection of bladder tumour (TURBT) (4). While there are a range of investigative modalities to aid in diagnosis of bladder cancer, TURBT remains to be the cornerstone for diagnosis and treatment of bladder cancer. It allows to assess the local staging and grading of the tumour as well as relieving symptoms if present. It will provide information that will guide us to arrange the appropriate next line of investigations or therapy. Various guidelines emphasize the key role of TURBT, in particular complete resection of all visible tumours when technically safe and feasible. Carcinoma in situ (CIS) is an exception, however, because it is often widespread or even inconspicuous with no definite demarcation of the tumour margins. Hence the role of endoscopic assessment and resection is to establish the diagnosis so that intravesical therapy can be instituted if deemed appropriate. 


\section{Preoperative considerations}

It is important to take a focussed history pertaining to haematuria, associated lower urinary tract symptoms, and risk factors of urothelial cancer such as smoking, occupational exposure, chronic inflammation/infection, history of pelvic radiation. Physical examination would entail the assessment of body habitus and abdomen and genitalia as well as digital rectal examination to assess the prostate in men. Investigations would include, but not limited to, basic blood and urine tests including cytology if high risk factors. Computed tomography intravenous pyelogram (CT IVP) is the imaging modality of choice to assess upper tract pathology. More recently, two metaanalyses calculated sensitivity of $87 \%$ and $92 \%$ and specificity of $79 \%$ and $87 \%$, respectively, in distinguishing NMIBC from muscle invasive bladder cancer (MIBC), with multiparametric magnetic resonance imaging (mpMRI) $(5,6)$. Although mpMRI of the Bladder before TURBT has been shown to differentiate MIBC and NMIBC (7) using Vesical Imaging-Reporting and Data System (VI-RADS), cystoscopy and bladder tumour resection remains the gold standard for diagnosis and initial management of bladder cancer. Bimanual examination is also still recommended by the guidelines, for clinical staging of bladder tumour, although its routine use is debated unless it appears invasive. The preoperative assessment should not only facilitate the assessment of risk of malignancy but ensure appropriate investigations are completed in order for patients to undergo TURBT safely under spinal or general anaesthetics. Furthermore, it would also minimise the risk of adverse events and avoid unnecessary overzealous resection in the setting of situations that are not aimed to cure (i.e., metastatic or palliative cancer).

\section{Factors that would affect recurrence and progression}

Bladder cancer is associated with a high rate of recurrence among patients with NMIBC. A number of studies assessed the factors that might be associated with recurrence and progression of bladder cancer following TURBT. The majority of the studies were rated as low quality and no strong recommendations were made in the guidelines due to study heterogeneity and hence low grade evidence. Nevertheless, there were a number of factors that have been shown to significantly influence the outcomes.

The largest randomised controlled trial (RCT) included 2,596 patients in seven European Organization for
Research and Treatment of Cancer (EORTC) trials. It demonstrated recurrence rates ranging from $15 \%$ to $61 \%$ and progression rates of less than $1 \%$ to $17 \%$ at one year. At five years, the probabilities of recurrence and progression ranged from $31 \%$ to $78 \%$ and from less than $1 \%$ to $45 \%$, respectively. The risk of recurrence was linked to a number of clinicopathologic factors including size, multifocality, prior recurrence, stage, CIS and grade (8). Based on these key factors the European Organization for Research and Treatment of Cancer (EORTC) scoring system and risk tables for recurrence and progression in patients with NMIBC have been developed as shown in Tables 1-3 (8).

The main drawbacks of the above EORTC tables were that most patients received intravesical chemotherapy and did not undergo second TURBT. Patients with CIS alone were also excluded. Subsequently the Spanish Urological Oncology Group (CUETO) developed a scoring model for Bacillus Calmette-Guérin (BCG)-treated patients that predict the short- and long-term risks of recurrence and progression (9) (Table 4). It showed that EORTC risk tables overestimated the risk of recurrence and progression in high risk patients likely due to a low percentage of patients receiving intravesical BCG.

More recently, prognostic factors were evaluated in NMIBC patients treated with 1-3 years of BCG therapy. NMIBC patients at high risk of recurrence and progression did poorly despite maintenance BCG. However, it was limited by lack of repeat TURBT and exclusion of CIS (10).

\section{TURBT quality and its influence on outcomes}

In addition to the above individual patient and tumour characteristics 'good quality' TURBT is of paramount importance as incomplete TURBT could lead to a higher rate of upstaging and inferior oncological outcomes. One of the key determinants at the time of TURBT is whether detrusor muscle was obtained. All the guidelines (EAU, NICE, NCCN and AUA) highlighted the importance of fully resecting all visible tumour and obtaining muscle at the time of TURBT particularly when high-grade disease is initially identified and, if no muscle was contained in the specimen, a further resection is recommended.

Chamie et al. conducted a retrospective study on 1,865 patients who underwent TURBT recorded in the Surveillance, Epidemiology and End Results (SEER) registry. They found that detrusor was reported to be present in only $52 \%$ of cases, irrespective of stage or grade. The absence of muscle translated into an adverse impact on five-year mortality (11). 
Table 1 Weighting used to calculate disease recurrence and progression scores

\begin{tabular}{|c|c|c|}
\hline Factor & Recurrence & Progression \\
\hline \multicolumn{3}{|l|}{ Number of tumours } \\
\hline Single & 0 & 0 \\
\hline $2-7$ & 3 & 3 \\
\hline$\geq 8$ & 6 & 3 \\
\hline \multicolumn{3}{|l|}{ Tumour diameter } \\
\hline$<3 \mathrm{~cm}$ & 0 & 0 \\
\hline$\geq 3 \mathrm{~cm}$ & 3 & 3 \\
\hline \multicolumn{3}{|l|}{ Prior recurrence rate } \\
\hline Primary & 0 & 0 \\
\hline$\leq 1$ recurrence/year & 2 & 2 \\
\hline$>1$ recurrence/year & 4 & 2 \\
\hline \multicolumn{3}{|l|}{ Category } \\
\hline $\mathrm{Ta}$ & 0 & 0 \\
\hline $\mathrm{T} 1$ & 1 & 4 \\
\hline \multicolumn{3}{|l|}{ Concurrent CIS } \\
\hline No & 0 & 0 \\
\hline Yes & 1 & 6 \\
\hline \multicolumn{3}{|l|}{ Grade } \\
\hline G1 & 0 & 0 \\
\hline G2 & 1 & 0 \\
\hline G3 & 2 & 5 \\
\hline Total score & $0-17$ & $0-23$ \\
\hline
\end{tabular}

Mariappan et al. evaluated the outcomes following TURBT on two Scottish cohorts with bladder cancer (12). They showed a significant reduction in the recurrence rate at first cystoscopy following TURBT based on the presence of detrusor muscle in the specimen. $(43 \%$ and $70 \%$ if muscle was absent compared to $18 \%$ and $39 \%$ if muscle was present). The presence of detrusor muscle in the specimen is considered as a surrogate for the resection quality except in low grade or non-invasive tumours.

\section{Second TURBT (re-resection or re-look TURBT)}

Repeat resection should be performed for any high grade or
Table 2 Probability of recurrence according to total score

\begin{tabular}{lccccc}
\hline \multirow{2}{*}{$\begin{array}{l}\text { Recurrence } \\
\text { score }\end{array}$} & \multicolumn{2}{c}{$\begin{array}{c}\text { Probability of } \\
\text { recurrence at 1 year }\end{array}$} & & \multicolumn{2}{c}{$\begin{array}{c}\text { Probability of } \\
\text { recurrence at 5 years }\end{array}$} \\
\cline { 2 - 3 } \cline { 5 - 6 } & $\%$ & $95 \% \mathrm{Cl}$ & & $\%$ & $95 \% \mathrm{Cl}$ \\
\hline 0 & 15 & $10-19$ & & 31 & $24-37$ \\
$1-4$ & 24 & $21-26$ & & 46 & $42-49$ \\
$5-9$ & 38 & $35-41$ & & 62 & $58-65$ \\
$10-17$ & 61 & $55-67$ & & 78 & $73-84$ \\
\hline
\end{tabular}

Table 3 Probability of disease progression according to total score

\begin{tabular}{lccccc}
\hline \multirow{2}{*}{$\begin{array}{l}\text { Progression } \\
\text { score }\end{array}$} & \multicolumn{2}{c}{$\begin{array}{c}\text { Probability of } \\
\text { progression at 1 year }\end{array}$} & & \multicolumn{2}{c}{$\begin{array}{c}\text { Probability of } \\
\text { progression at 5 years }\end{array}$} \\
\cline { 2 - 3 } \cline { 5 - 6 } & $\%$ & $95 \% \mathrm{Cl}$ & & $\%$ & $95 \% \mathrm{Cl}$ \\
\hline 0 & 0.2 & $0-0.7$ & & 0.8 & $0-1.7$ \\
$2-6$ & 1 & $0.4-1.6$ & & 6 & $5-8$ \\
$7-13$ & 5 & $4-7$ & & 17 & $14-20$ \\
$14-23$ & 17 & $10-24$ & & 45 & $35-55$ \\
\hline
\end{tabular}

tumour involving the lamina propria (T1), or incompletely resected tumour, especially when detrusor muscle was not identified in the resected specimens (13). Despite further experience and education on the significance of high grade or $\mathrm{T} 1$ disease residual tumour was found in $17-72 \%$ of Ta and $33-78 \%$ of $\mathrm{T} 1$ tumours (14-18). In addition, the risk of under staging NMIBC is significant, with $15-30 \%$ of cases being upstaged to T2 at the second TURBT (17-19). The risk of upstaging $(\geq \mathrm{T} 2)$ increases up to $49 \%$ in patients with $\mathrm{T} 1$ disease if no muscle was obtained in the initial specimen (20).

A RCT conducted by Divrik et al. also showed improved oncological outcomes in patients with $\mathrm{T} 1$ disease who underwent second TURBT (21). Recurrence-free survival (RFS), progression-free survival (PFS) and cancer-specific survival (CSS) were $52 \%$ vs. $21 \%(\mathrm{P}=0.0001), 93 \%$ vs. $76 \%$ $(\mathrm{P}=0.0001)$ and $83.3 \%$ vs. $68.6 \%(\mathrm{P}=0.038)$, respectively.

Similarly, Tseng et al. demonstrated improved RFS and PFS following second TURBT in patients with T1 disease (22). The 2-year RFS rates were $74.6 \%$ and $60 \%$ and the PFS rates were $91.2 \%$ and $87.5 \%$ in groups 1 and 2, respectively (23). These results emphasize the importance of second TURBT in T1 bladder cancer not only for restaging but also for therapeutic benefit (24). Improved CCS and overall survival (OS) were also 
Table 4 Weighting used to calculate recurrence and progression scores

\begin{tabular}{lcc}
\hline Factor & Recurrence score & Progression score \\
\hline Gender & 0 & 0 \\
M & 3 & 0 \\
F & & \\
Age (years) & 0 & 0 \\
$<60$ & 1 & 0 \\
$60-70$ & 2 & 2 \\
$>70$ &
\end{tabular}

Recurrent tumour

$\begin{array}{lll}\text { No } & 0 & 0 \\ \text { Yes } & 4 & 2\end{array}$

No. of tumours

$\begin{array}{lll}\leq 3 & 0 & 0 \\ >3 & 2 & 1\end{array}$

T category

Ta

$\mathrm{T} 1$

Associated CIS

\begin{tabular}{ccc} 
No & 0 & 0 \\
Yes & 2 & 1 \\
Grade & & \\
G1 & 0 & 0 \\
G2 & 1 & 2 \\
G3 & 3 & 6 \\
Total scores & $0-16$ & $0-14$ \\
\hline
\end{tabular}

CIS, carcinoma in situ.

demonstrated (25).

Although the rate of upstaging is significantly lower in patients with $\mathrm{T} 1$ disease in the presence of muscle in the initial resection, second TURBT is still recommended due to a potential for progression in $1-14 \%$ of cases $(18,20)$. It would help achieve a higher rate of clear resection, more accurate staging, and improved treatment selection (i.e. immediate radical cystectomy $v s$. intravesical therapy) and prognostication. While the majority of the studies showed that the greatest benefit from reTURBT was achieved in patients with T1 disease, patients with Ta high grade tumours fared the worst in terms of recurrence and progression rates, highlighting the significance of high grade disease regardless of its depth of invasion (26).

Therefore, all guidelines (AUA, EAU, NCCN and NICE) recommend re-TURBT for these indications within 2-6 weeks due to the high incidence of residual tumour and understaging (27). It is critical to resect all visible papillary tumours, especially T1 lesions, as intravesical BCG is used to treat CIS and does not treat residual T1 papillary tumours.

However, Gontero et al. showed that, while second TURBT had a positive impact on recurrence, progression, CSS and OS in the presence of muscle in the primary specimen, it did not improve the outcome for any of the oncological endpoints (28). It is also important to consider possible impact on potential complications, resource allocation and healthcare costs that are associated with second TURBT. With ongoing technical refinements and advances second TURBT might not be necessary in all of these patients in the future. We would require further high quality studies which could identify factors that could be used as a predictive tool for better selection of patients for second TURBT while avoiding an unnecessary surgery with possible risks and costs (29).

\section{Factors that affect resection quality ('Good quality' TURBT)}

\section{Surgeon experience}

Studies showed that resection quality depends on surgeon experience. It is influenced by education and experience of surgeon as well as surgical resection technique. Although it is perceived to be relatively straightforward endoscopic procedure, satisfactory resection of bladder tumours requires significant technical skill and 3-dimentional orientation skills to achieve accurate pathologic diagnosis.

A retrospective review of 463 TURBTs demonstrated there was a significantly less likelihood of containing detrusor muscle among high-risk patients when TURBT was performed by residents $(\mathrm{P}=0.006)$ (30). Huang et al. reported similar findings (31). These studies were in agreement with Mariappan et al., who demonstrated surgeon experience (year 5 trainee or higher) to be independently associated with the presence of detrusor muscle in TURBT specimen. Both the absence of detrusor muscle and resection by junior surgeons were associated with an increased recurrence rate at first followup cystoscopy (12). Rouprêt and colleagues evaluated 340 patients with T1 bladder cancer and corroborated the above findings (32). The detrusor muscle was found in $73 \%$ 


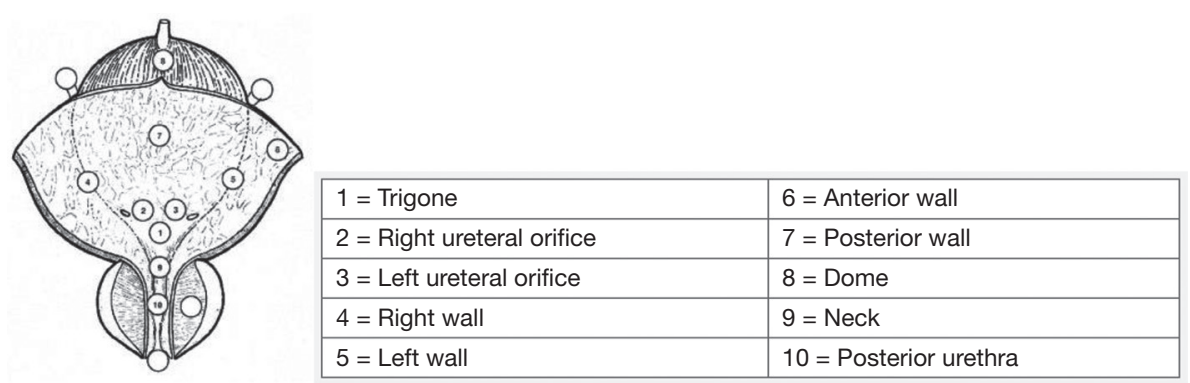

Figure 1 Bladder diagram [permission obtained from the EAU guidelines (36)].

of cases undertaken by senior surgeons compared to $61 \%$ by junior surgeons $(\mathrm{P}=0.02)$. There was $31 \%$ recurrence rate at first cystoscopy if detrusor was present and $53 \%$ if it was absent $(\mathrm{P}=0.01)$. They also showed that recurrence rate was independently associated with junior surgeons, regardless of the presence of detrusor muscle. Herr and Donat further supported this conclusion and proposed that fractionated wide resection of all tumours and re-resection could be used to improve the quality of TURBT (20). Complete resection with detrusor present, carried out by experienced surgeons, would be considered to be an essential benchmark for TURBT.

Conversely, Shoshany et al. found no significant difference in the presence of detrusor muscle based on surgeon experience (33). This discrepancy in the rate of detrusor muscle based on surgeon seniority would indicate several factors such as training environment, supervision, and educational techniques. While it is imperative to maintain high quality TURBT for optimal patient outcomes with improved efficacy and efficiency it must be balanced with further opportunity for quality surgical education and training for trainee surgeons. Of many proposed avenues simulation training model has been widely utilized in a number of surgical procedures. Although there are several proposed simulation programs that have been studied, there is a need for the development of validated simulation program that is associated with excellent transferability of skills to good quality TURBT. This will need to be incorporated into standard training curriculum (34).

Another more practical approach was suggested by Zainfeld and colleagues. They proposed an "intermittent resection technique". There was no increase in cautery artefact or decreased quality of resections. It would allow trainees to perform resection using short intermittent cuts rather than single large swipes. This would provide an enhanced opportunity for feedback to trainees which will enable them to adjust depth or direction of resection without compromising outcomes. There was no increase in cautery artefact or decreased quality of resections (35).

In addition to surgeon's experience a number of measures have been developed to achieve more satisfactory TURBT. It begins with the implementation of a perioperative bladder tumour checklist and bladder diagram (Figure 1) (16,36). A checklist can be used to ensure that all of the necessary equipment is available and thus avoid delays while the patient is under anaesthesia. The use of a bladder diagram was developed to document the location of all tumours and could lower recurrence rate (37). Jurewicz et al. also proposed to incorporate clinical photograph into operation report (16).

\section{Key steps and principles of TURBT}

After induction of anaesthesia patient is placed in the dorsal lithotomy position. The procedure can be performed either under spinal or general anaesthetics. General anaesthetics would be preferred if neuromuscular blockade is indicated to prevent stimulation of obturator nerve reflex (ONR) during the resection of lateral wall tumours. Bimanual examination of the bladder under anaesthesia is performed before resection. The prostate should also be assessed for men. The TURBT is undertaken after a detailed pancystoscopic evaluation using both 30- and 70-degree lenses for optimal assessment of the urethra, prostate and bladder including the bladder neck. Alternatively, a retroflexion manoeuvre using a flexible cystoscope can also be utilized. The anterior wall and the dome of the bladder might require suprapubic pressure for better visualisation.

If indicated a barbotage urine sample using normal saline irrigation can be obtained after inspection of the bladder. It is important not to overfill the bladder during tumour resection to avoid inadvertent perforation. Bimanual examination of the bladder under anaesthesia is also performed after resection for staging. Thickening of bladder wall might indicate MIBC, 
while palpable or mobile mass could suggest $\mathrm{cT} 3$ disease and fixed mass cT4 disease. Complete eradication of tumour is the key step of TURBT. If there are widespread red velvety flat lesions suggestive of CIS it would not be prudent to resect the entire lesions but instead selective biopsy would suffice with a view to intravesical therapy. If muscle is not confidently included in the specimen or concerned about cautery artefact cold cup biopsy of the base could also be performed to ensure accurate staging and the absence of invasion to detrusor muscle. Conventionally, large tumours are resected in a piecemeal fashion. This staged resection is often performed in a series of different layers until detrusor muscle is obtained. Judicious use of cautery would be employed to avoid potential cautery artefact on the specimen which would make it difficult for pathological interpretation of grading or staging of tumour.

\section{Complications of TURBT}

Although TURBT has been the technique of choice over decades with relatively low morbidity, it is far from perfect due to risk of complications as well as compromise in oncological outcomes from thermal damage to specimen margin, the absence of detrusor, tumour seeding, incomplete resection, and subsequently inaccurate pathological assessment. Complication rates are approximately $4-6 \%$ of which urinary tract infections and significant haematuria are most common. Bladder perforation and ONR are major complications and every measure should be taken to avoid these serious complications from both anaesthesia and surgery.

\section{Challenging situations}

\section{Tumours at the ureteral orifices}

Coagulation close to the ureteral orifices should be avoided as it may cause scarring and lead to ureteric obstruction (38). However, tumours that involve the ureteral orifices can be resected judiciously under pure cutting settings. This would be particularly beneficial as satisfactory renal function can be facilitated if cisplatinbased neoadjuvant chemotherapy is considered for MIBC. Although it may result in vesicoureteric reflux and would be uncommon to cause stricture, a temporary ureteric stent placement between 2 and 6 weeks can further reduce the risk. A form of imaging such as renal ultrasound, CT urogram or Diethylenetriamine Pentaacetic Acid (DTPA) renal scan after resection is recommended.

\section{Tumours on the lateral and anterior walls}

Resection of lateral wall tumours may result in stimulation of ONR resulting in increased risk of perforation (39). Strategies that have been shown to reduce the likelihood of ONR would include (I) avoidance of bladder overfilling, (II) reduced cutting current, (III) use of short intermittent burst current ('Stacatto'), (IV) use of bipolar electrocautery, and (V) use of neuromuscular blockade (40).

Tumours on the anterior wall could be challenging to resect and may require suprapubic depression by an assistant as well as proper resectoscope angles. More effective resection might be achieved by using open-angled loops.

\section{Tumours in bladder diverticulae}

Tumours within bladder diverticula are also considered to be difficult to obtain complete clearance due to suboptimal access and resection in particular if the diverticular neck is narrow and lack of muscularis propria layer. This would pose an increased risk of perforation and the inherent limitations in the assessment of the depth of tumour invasion. In view of this, while small, low grade tumours can be carefully resected, diverticulectomy, partial or radical cystectomy is considered to deal with large, high grade tumours in the diverticulum (38).

\section{Random biopsy}

Random bladder biopsy usually involves removal of bladder specimens with a cold cup forceps, sampling mostly mucosa and lamina propria layers with the intention to identify CIS in grossly normal-appearing areas. There is no strong evidence to support the routine use. Its main indications are in patients with negative cystoscopy but positive cytology, or with a history of high grade and nonpapillary tumours to further investigate the presence or the extent of CIS involvement. Biopsies should be taken from the trigone, dome, right, left, anterior and posterior bladder wall according to the EAU guidelines (41). This method of biopsy is therefore also known as site-directed biopsy. The incidence of positive random biopsies in areas of normal mucosa ranges from $1.5 \%$ to $14.5 \%$ which seem to influence management in $8 \%$ of patients $(41,42)$. Additionally, photodynamic diagnosis (PDD) or narrow band imaging-based cystoscopy have shown promising results and may substitute some of the indications for random biopsy, although no high level evidence is available to date. These enhanced imaging technologies will be discussed in details in the later section of this review article. 


\section{Prostatic urethra biopsy}

Prostatic urethra biopsy could also serve as an important aspect of staging and should be performed in patients with negative cystoscopy but positive cytology or in patients with CIS to assess its extent. The incidence of CIS was noted to be $11.7 \%$ in patients with T1 high grade urothelial cancer of the bladder (43). It could be very superficial and confined to the urethra but extend more deeply into ducts, acini or stroma of the gland. The risk of prostatic urethral involvement increases with higher stage, grade, bladder tumour located at the trigone or bladder neck and multiple tumours (44). If prostatic urethral biopsy is not performed in the first resection it should be included in the subsequent biopsy. Precollicular areas carry the largest concentration of prostatic ducts and hence should be obtained with the resection loop at 5 and 7 o'clock positions. Cold cup biopsy can be employed if superficial disease only is suspected. If there is confirmed presence of CIS or T1 disease in the prostatic urethra, these patients should undergo a formal transurethral resection of the prostate (TURP) for more accurate staging, and so they can get maximal benefit from intravesical BCG therapy. More recently, advances in enhanced cystoscopic imaging technologies in the form of fluorescence cystoscopy for PDD, narrow-band imaging (NBI) have shown more accurate assessment and treatment of NMIBC. En bloc resection of bladder tumour technique (EBRT) has also been gaining increasing popularity due to its ability to include detrusor muscle, with less risk of seeding and complications. Further details are discussed in another section.

\section{Recent technological advances to improve outcomes}

\section{Bipolar}

TURBT has been performed effectively using monopolar electrocautery since its introduction in the early 1900s (45). More recently, with advances in bipolar technology, it has been widely adopted for TURP. One of the main advantages is that it obviates the need for hypotonic solution such as water or glycine as a fluid medium during TURP. Instead it requires an isotonic fluid (e.g., Normal saline) for effective resection and haemostasis and has virtually eliminated the risk of TUR syndrome. In addition due to its inherent property the current only travels between the resection loop and resectoscope sheath allowing for lower energy systems and theoretically reduces the risk of ONR as a result.
Given the above perceived improved safety profile bipolar technology has extended to TURBT.

Safety was also demonstrated by Puppo et al. who reported a very low rate of complications after 1,000 cases were performed with bipolar technology (46). Pathologic analysis of specimens is essential for the correct assignment of tumour grade and stage. Wang et al. were the first group who reported the usage of bipolar TURBT and compared 11 patients who underwent bipolar TURBT with a matched cohort of 11 patients who underwent monopolar TURBT. Although tissue chips were smaller due to smaller bipolar resection loop, there was no difference in the degree of cautery artefact between the two groups, and a proper diagnosis was achieved in all cases (47). Yang et al. also showed no difference in the grade of thermal damage between the two groups (48). Venkatramani et al. reported a significantly lower incidence of cautery artefact in the bipolar arm in their large, prospective randomised trial $(25 \%$ vs. $46.7 \%, \mathrm{P}=0.0096$ ) (49).

There was also less decline in postoperative haemoglobin and shorter duration of catheterization in the bipolar TURBT group (48). Furthermore, reducing the power settings to $50-\mathrm{W}$ cutting and $40-\mathrm{W}$ coagulation may reduce the incidence of ONR and bladder perforation to negligible risk while still maintaining diagnostic and therapeutic efficacy (50). These findings were supported by prospective studies. Equivalence was demonstrated between the two techniques in terms of complications including thermal effects, ONR, bladder perforation, postoperative haemoglobin and sodium levels $(49,51,52)$. Xishuang et al. compared outcomes among monopolar, plasmakinetic (bipolar), and holmium laser in the management of NMIBC. Bipolar and holmium laser groups had significantly decreased the occurrence of ONR, bladder perforation, bladder irritation, postoperative irrigation, catheterisation time, and hospitalisation time without difference in recurrence rates at 2 years (53).

A recent meta-analysis including six RCTs found bipolar TURBT to be associated with shorter operative time, less blood loss, shorter catheterization time, decreased ONR, and bladder perforation in comparison to traditional monopolar procedures. In addition, a decreased rate of tumour recurrence was noted at 2 years (54). While the physiologic mechanisms of some of these results are unclear, little question remains regarding the safety profile of bipolar resection for bladder cancer. Teoh et al. conducted an RCT on monopolar versus bipolar TURBT. They found that bipolar TURBT was also significantly associated with 
higher rate of detrusor muscle sampling (55). Although the evidence is still controversial, bipolar TURBT appears to offer at least equivalent, if not superior, in a number of perioperative parameters and pathological outcomes.

\section{Enhanced cystoscopy}

\section{PDD (fluorescence cystoscopy)}

The management of NMIBC is expensive, stemming from high recurrence rates necessitating repeat TURBT and frequent surveillance cystoscopies. The key limitation in the endoscopic management of early-stage bladder cancer is the inherent difficulty of identifying all areas of malignancy within the bladder due to the multifocal nature of bladder cancer and often inconspicuous, yet significant, lesions characteristic of CIS with standard visualisation techniques. Advances in the field of TURBT have facilitated the detection of these bladder lesions and help achieve more satisfactory TURBT. One of the major innovations is the introduction of PDD.

Fluorescence cystoscopy, commonly termed bluelight cystoscopy (BLC), is the most validated technique for enhanced visualisation and could then reduce the rate of recurrence. It requires intravesical instillation of a photosensitizing agent, most commonly hexaminolevulinate (HAL) one hour prior to planned resection. It is absorbed preferentially by malignant cells. Tumour cells will fluoresce red with its distinct demarcation when illuminated by blueviolet light (380-480 nm) (35).

It has demonstrated superior tumour detection rate and resection with downstream effects on cancer outcomes and costs of care (56). Witjes et al. also examined costs over time with application of BLC finding a higher initial cost of BLC but decreased costs overall due to fewer repeat procedures required in light of less recurrence (57).

Prospective randomised controlled studies showed significantly improved detection rate $(16 \%)$ as well as lower recurrence rate $(47 \%$ vs. $56 \%, \mathrm{P}=0.026)$ and prolonged median time (16.4 vs. 9.4 months, $\mathrm{P}=0.04$ ) to recurrence compared to white light cystoscopy (WLC) $(58,59)$.

A meta-analysis reviewed prospective studies on 1,345 patients and demonstrated HAL BLC detected significantly more $\mathrm{Ta} / \mathrm{T} 1$ tumours $(14.7 \% ; \mathrm{P}<0.001)$ and CIS lesions $(40.8 \% ; \mathrm{P}<0.001)$ than WLC. In $26.7 \%$ of patients, CIS was detected only by $\mathrm{BLC}(\mathrm{P}<0.001)$ and was also significant in patients with primary $(28.0 \% ; \mathrm{P}<0.001)$ and recurrent cancer $(25.0 \% ; \mathrm{P}<0.001)$. Furthermore, recurrence rates up to 12 months were significantly lower overall with $\mathrm{BLC}$ versus WLC (34.5\% vs. 45.4\%; $\mathrm{P}=0.006)$ (60).
A systematic review and meta-analysis compared 644 who underwent BLC- and 657 WLC-based TURBT. It showed a significantly lower rate of progression in patients treated with BLC- vs. WLC. (6.8\% vs. 10.7\%, $\mathrm{P}=0.01)$ (61). More recently, a RCT has also shown a reduction in recurrence and progression with fluorescence guided TURBT (62).

Despite the several advantages PDD had lower specificity than WLC (63\% vs. 81\%) (63). False-positivity could arise from inflammation or recent resection or BCG instillation. However, recent US prospective multicentre studies showed equivalent false-positive rate of $9.1 \%$ for both groups treated with WLC and BLC $(64,65)$.

Another photosensitizing agent, 5-ALA is not currently approved for routine clinical use for the detection of bladder cancer in Europe or the United States but its role has been studied in a number of clinical trials. Inoue et al. evaluated the clinical value of PDD with intravesical and oral instillation of 5-ALA and PDD-guided TURBT for NMIBC in a multi-institutional retrospective study in 210 patients (66). 5-ALA-guided TURBT improved detection of CIS, as $72.1 \%$ of flat lesions could only be detected with this technology. The route of administration of 5-ALA did not affect diagnostic accuracy or RFS.

A systematic review and analysis of 14 RCTs including 2,906 patients (6 5-ALA and 9-HAL) demonstrated a decreased risk of short- and long-term recurrence. However, there was no difference in progression and mortality rates and should be interpreted with caution due to inconsistency between trials and potential susceptibility to performance and publication bias (67).

\section{Narrow band imaging}

Alternative to fluorescence cystoscopy, narrow band imaging (NBI) has emerged as a viable tool for improved tumour visualisation that avoids preoperative bladder instillation of a photosensitizing agent. NBI technology relies on the hypervascular nature of bladder cancer to aid in differentiation of normal urothelium. Optical filters reduce light to blue $(415 \mathrm{~nm})$ and green $(540 \mathrm{~nm})$ components which are preferentially absorbed by haemoglobin in neoplastic cells providing enhanced contrast between vascularized cancer tissue and normal urothelium. NBI, though not as extensively studied or validated as BLC, has been demonstrated to similarly improve tumour detection and in some series, RFS rates. A recent metaanalysis evaluated the diagnostic accuracy of NBI-assisted cystoscopy compared with WLC for NMIBC (68). NBI detected tumours in an additional $17 \%$ of patients and 
found an additional $24 \%$ of tumours compared with WLC. No difference in the false-positive rate of tumour detection was noted between NBI and WLC.

In a meta-analysis of eight clinical trials involving around 1,000 patients NBI had increased sensitivity over WLC (94\% vs. $85 \%$, respectively), with similar specificity ( $85 \%$ vs. $87 \%$, respectively) for the detection of any NMIBC; for CIS detection, the sensitivity and specificity of NBI were $93 \%$ and $77 \%$, respectively (69). A RCT compared patients with NMIBC undergoing NBI or WLC and found the NBI group had a reduced recurrence rate at 1 year $(32.9 \%$ vs. $51.4 \%, \mathrm{OR}=0.62 ; \mathrm{P}=0.0141)(70)$. Another RCT showed no significant difference overall $(27.1 \%$ white light and $25.4 \%$ NBI; $\mathrm{P}=0.585$ ), recurrence rate was lower at three and twelve months for low-risk tumours (5.6\% vs. $27.3 \%$; $\mathrm{P}=0.039)$ (71).

Drejer et al. compared WLC, BLC and NBI in detection of CIS or flat dysplasia. When analysed at patient level, NBI and BLC had a significantly higher sensitivity compared with WLC (NBI: 95.7\%, PDD: 95.7\% vs. WL: 65.2\%, $\mathrm{P}<0.05)$, whereas specificity was not significantly different between the 3 methods (NBI: $52.0 \%$, PDD: $48.0 \%$, and WL: $56.8 \%)$. NBI and PDD also had a significantly higher sensitivity than WLC (NBI: $72.7 \%$ and PDD: $78.2 \%$ vs. WLC: $52.7 \%, \mathrm{P}<0.05)$ per biopsy, whereas the positive predictive values were not significantly different (NBI: 23.7\%, PDD: $22.2 \%$, and WLC: $19.0 \%$ ). A network metaanalysis found no significant difference between NBI and BLC in terms of reduced recurrence risk compared with WLC (72). However, the two methods have not been directly compared in clinical trials.

NBI was found to be a valid alternative to PDD regarding diagnosis of CIS and flat dysplasia (73). Further study of NBI will better define its role and potential advantages in the management of bladder cancer (72).

Enhanced cystoscopy using PDD or NBI represents a tremendous advance in the management of NMIBC and appears to be an effective adjunct to WLC as this might allow more patients at risk of recurrence and progression to be treated in a timely fashion. However, due to a lack of long term mature clinical data it would not adequately address an issue with understaging.

\section{Enhancing the quality of transurethral resection: the importance of a complete TURBT and the en bloc resection}

While TURBT has been the most widely used technique for resection of bladder tumour over several decades, it has been fraught with multiple concerns that need to be addressed. The resection is performed in a piecemeal fashion also known as 'incise and scattering' technique. Essentially it is against oncological principles as it will breach surgical margins and increase the risk of potential seeding and implantation by tumour fragmentation. This could then affect oncological outcomes with potentially higher rate of recurrence and progression. There is also a risk of perioperative morbidities such as ONR, and bladder perforation. Furthermore, accurate histological assessment might not be possible due to fragmentation and cautery artefact as well as high rate of incomplete resection or absent detrusor muscle. Due to relatively high risk of recurrence and progression there is a need for improved accuracy and novel resection techniques have been developed with encouraging results. The one of the most notable technical advances in endoscopic management of bladder tumours in the last decade is en bloc resection of bladder tumour (EBRT) as an alternative to conventional TURBT (74). It may overcome these shortcomings, representing the best surgical technique to perform a "Good-quality TURBT".

Theoretically, ERBT offers three goals: to improve the resection quality, lowering perioperative complication rates, and decreasing recurrence rates. It allows more accurate pathological assessment by preserving the orientation of the tumour, respecting margin and depth adhering to sound oncological principles. Kawada et al. were the first group that presented a case report on ERBT using a rotational resection technique that is still the conventional method of choice to date (75). The presence of detrusor is one of the principal surrogate markers for the quality and completeness of the resection (12) and a number of studies showed high quality resected specimens with the presence of detrusor muscle in $96-100 \%$ of cases. Furthermore, it would lead to potentially less risk of tumour shedding and implantation as well as less perioperative complications (i.e., less bleeding, ONR, catheterisation, hospitalisation).

\section{En bloc resection of bladder tumour (EBRT) technique}

Typically a circumferential incision is made on the mucosa around the tumour with the tip of laser fibre maintaining a distance of $5-10 \mathrm{~mm}$ from tumour edge. The dissection is carried out in the macroscopically normal mucosa and extended through the submucosal and muscular layer. The muscular fibres are divided cautiously from the periphery to the centre of the tumour. The lesion is then detached and removed using various exit strategies (i.e., Ellick evacuator, 
endobag or basket or grasper). Alternatively, ERBT may be performed by undermining the tumour base via antegrade application of short energy impulses. In general $30 \%$ of patients might not be suitable for EBRT due to tumour size and location. Although successful resection of tumour up to $7.5 \mathrm{~cm}$ has been reported (76), it is often avoided if tumour is greater than $3 \mathrm{~cm}$ in size or arises from anterior or posterior bladder wall (77).

This can be performed using various types of energy source: laser (Thulium, KTP, Holmium), Hybrid-Knife, electrode (bipolar, monopolar electric current).

KTP laser has been shown to be effective using $30 \mathrm{~W}$ but due to its side fire property and its wider penetration of tissue fulguration compared to Ho:YAG or Tm:YAG laser it could be associated with a greater risk of damage out of sight. This would make Ho:YAG and Tm:YAG more suitable form of laser for this procedure. The Tm:YAG laser mostly uses $30-50 \mathrm{~W}$ and is the most adopted technology to date (74).

Electrical current mode as an energy source also has shown equivalent results. Sureka et al. used Tungsten loop electrode and monopolar cautery (78). Hurle used J electrode (Collins knife) and monopolar cautery (79). Zhang et al. also described retrograde ERBT using the same loop electrode and was able to include detrusor muscle in $100 \%$ of cases in the resection specimen and the base of the tumour compared to $54 \%$ and $70 \%$ of conventional TURBT specimens. There was no difference in operative time, or complication rates or recurrence rates (80). Bipolar button electrode was also utilized to perform ERBT. No intraoperative complications were noted and detrusor muscle was present in all cases (81).

Hydrodissection via needle or waterjet may further facilitate resection through the right layer and thereby help achieve negative margin (82). This technique has been widely utilized in endoscopic gastrointestinal procedures to achieve the removal of the tumour in its entirety with the aim of improved pathological specimen and potentially superior cancer outcomes and avoiding repeat procedures in select cases. A RCT showed superiority of Hydrodissection technique with regard to the number of histopathologically confirmed complete resections ( $57 \%$ vs. $9 \%, \mathrm{P}<0.001)$ in comparison to TURBT (83).

A meta-analysis demonstrated outcomes in favour of ERBT over the conventional TURBT. The perioperative parameters were significantly superior in the ERBT group (i.e., Bladder perforation, ONR, hospitalisation and catheterisation time). Furthermore, recurrence rate at two years was significantly lower $(\mathrm{OR}=0.66,95 \%$
CI: 0.47-0.92, $\mathrm{P}=0.02)(84)$. Another meta-analysis included 13 trials comparing laser enucleation of bladder tumour versus TURBT and confirmed the superior properties of ERBT in all of the above parameters (85).

A systematic review reported $1 \%$ risk of grade III complications, mostly bladder perforation or bleeding. $6.5 \%$ were converted to conventional TURBT due to unfavourable tumour location or failure to satisfactorily extract the specimen (86).

More recently Li et al. compared patients who underwent Tm:YAG laser ERBT and TURBT. In addition to shorter hospitalisation time and catheterisation time the Tm:YAG laser group was also associated with significantly shorter operation duration and postoperative irrigation time (68).

Kramer also compared electrical en bloc and laser ERBT. There was no significant difference between the two groups but higher rate of conversion to conventional TURBT in the former group (87). It appears that satisfactory specimen resection using ERBT technique is achieved regardless of the type of energy source.

An overview of ERBT studies is shown in Table 5.

Hermann and colleagues investigated the safety and effectiveness of diode laser technology without sedation in an outpatient setting. 21 patients with low grade bladder cancer underwent diode ablation and the median pain score was 1 using a visual analogue scale. 24\% had new Ta lowgrade recurrence within 13 months but no progression was identified. It was associated with a huge reduction in healthcare costs (102).

Due to perceived experience of prolonged operative time and limitations in regards to tumour size $<3 \mathrm{~cm}$ with EBRT, Hayashida et al. applied combined endoscopic mucosal resection using a polypectomy snare and EBRT technique (103). The measured outcomes were comparable including operation time, duration of catheterization, hospitalization, and recurrence rates with greater ability to make a correct diagnosis.

\section{Current and future perspectives}

ERBT seems to be a safe and effective resection technique. A new envisaged goal is also to decrease the number of second TURBTs. It can be facilitated by achieving higher rate of detrusor in the initial specimen and minimal tumour fragmentation using the correct ERBT technique. Large randomised prospective multicentre comparative trials are needed to clarify outcomes and to further assist in identifying suitable patients for this technique. This 


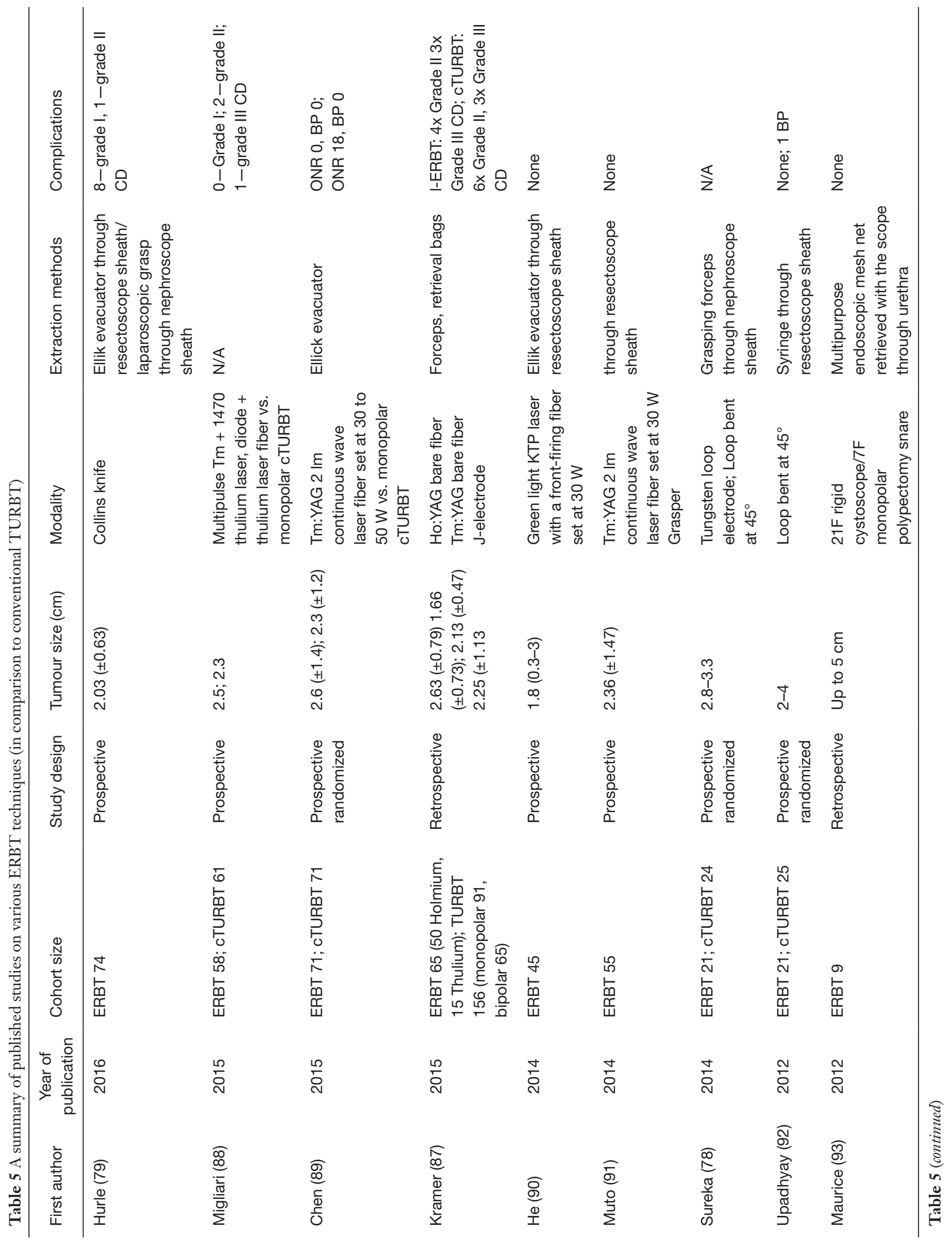




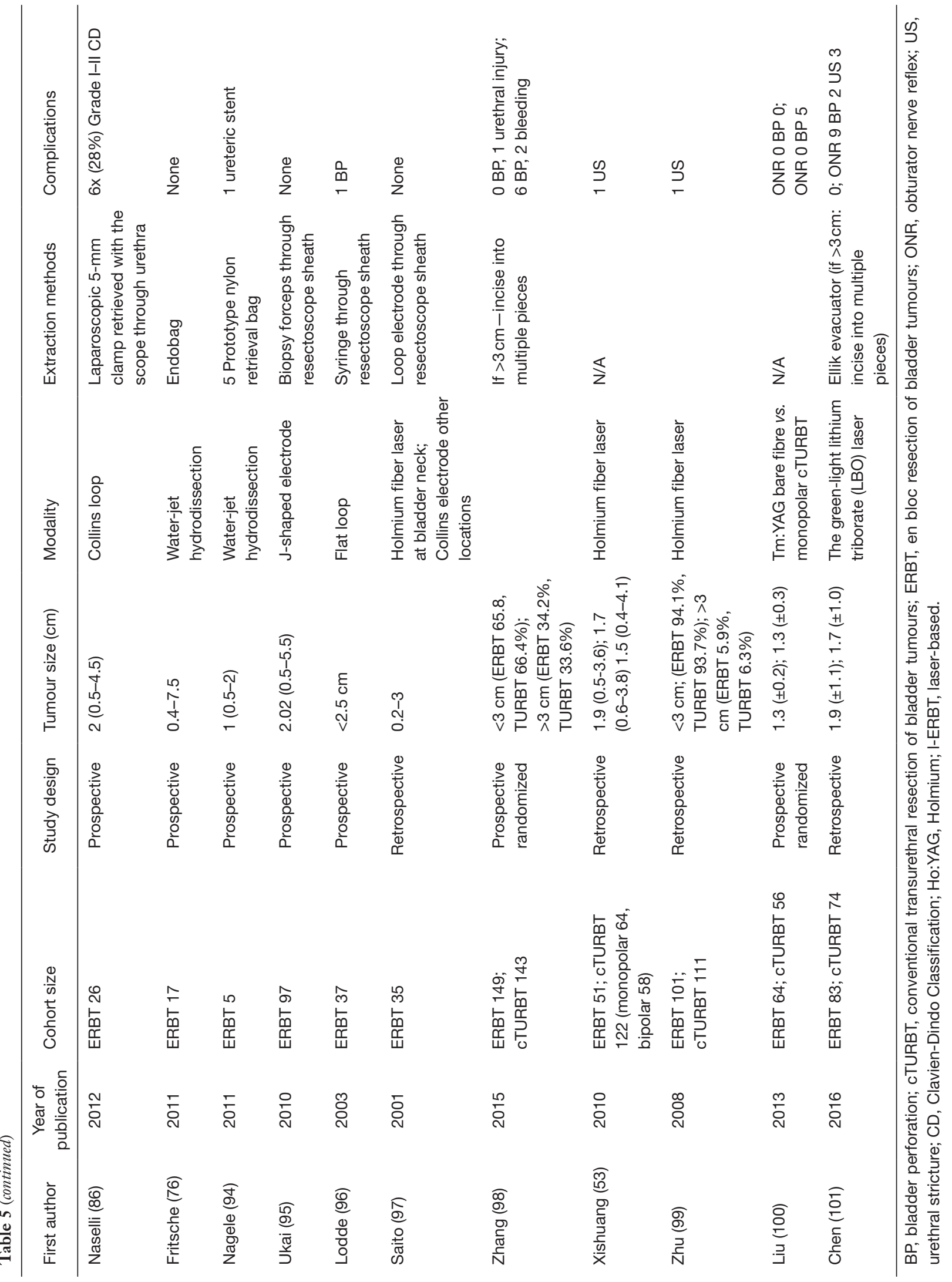


will enable us to offer more personalized approach to management of patients with bladder cancer. Moreover, it would be important to acquire appropriate instruments and dedicated surgical team as well as genitourinary pathologist who are familiar with the procedure.

\section{Conclusions}

TURBT remains a gold standard for the evaluation and management of bladder cancer. Quality of resection has a direct impact on patient outcomes. Conventional TURBT has several limitations. These include suboptimal pathological assessment, perioperative morbidities, in particular ONR and bladder perforation, and high recurrence and progression rates. Modifications in resection technique (i.e., ERBT) and enhanced cystoscopy in the form of PDD and NBI demonstrated promising results to address some of these inherent limitations of conventional TURBT for diagnosis and treatment of bladder cancer. Future seems to be promising but ongoing research will be required to further define the role of these novel techniques and technologies.

\section{Acknowledgments}

Funding: None.

\section{Footnote}

Provenance and Peer Review: This article was commissioned by the Guest Editor (Shomik Sengupta) for the series "Surgery for Urologic Cancers" published in Translational Andrology and Urology. The article has undergone external peer review.

Conflicts of Interest: Both authors have completed the ICMJE uniform disclosure form (available at http://dx.doi. org/10.21037/tau.2019.09.38). The series "Surgery for Urologic Cancers" was commissioned by the editorial office without any funding or sponsorship. The authors have no other conflicts of interest to declare.

Ethical Statement: The authors are accountable for all aspects of the work in ensuring that questions related to the accuracy or integrity of any part of the work are appropriately investigated and resolved.

Open Access Statement: This is an Open Access article distributed in accordance with the Creative Commons
Attribution-NonCommercial-NoDerivs 4.0 International License (CC BY-NC-ND 4.0), which permits the noncommercial replication and distribution of the article with the strict proviso that no changes or edits are made and the original work is properly cited (including links to both the formal publication through the relevant DOI and the license). See: https://creativecommons.org/licenses/by-nc-nd/4.0/.

\section{References}

1. Siegel RL, Miller KD, Jemal A. Cancer statistics, 2017. CA Cancer J Clin 2017;67:7-30.

2. Tan WS, Rodney S, Lamb B, et al. Management of nonmuscle invasive bladder cancer: A comprehensive analysis of guidelines from the United States, Europe and Asia. Cancer Treat Rev 2016;47:22-31.

3. Svatek RS, Hollenbeck BK, Holmang S, et al. The economics of bladder cancer: costs and considerations of caring for this disease. Eur Urol 2014;66:253-62.

4. Brausi M, Collette L, Kurth K, et al. Variability in the Recurrence Rate at First Follow-up Cystoscopy after TUR in Stage Ta T1 Transitional Cell Carcinoma of the Bladder: A Combined Analysis of Seven EORTC Studies. Eur Urol 2002;41:523-31.

5. Woo S, Suh CH, Kim SY, et al. Diagnostic performance of MRI for prediction of muscle-invasiveness of bladder cancer: A systematic review and meta-analysis. Eur J Radiol 2017;95:46-55.

6. Gandhi N, Krishna S, Booth CM, et al. Diagnostic accuracy of magnetic resonance imaging for tumour staging of bladder cancer: systematic review and metaanalysis. BJU Int 2018;122:744-53.

7. Panebianco V, Narumi Y, Barchetti G, et al. Should We Perform Multiparametric Magnetic Resonance Imaging of the Bladder Before Transurethral Resection of Bladder? Time to Reconsider the Rules. Eur Urol 2019;76:57-8.

8. Sylvester RJ, van der Meijden AP, Oosterlinck W, et al. Predicting recurrence and progression in individual patients with stage Ta T1 bladder cancer using EORTC risk tables: a combined analysis of 2596 patients from seven EORTC trials. Eur Urol 2006;49:466-5; discussion 475-7.

9. Fernandez-Gomez J, Madero R, Solsona E, et al. Predicting Nonmuscle Invasive Bladder Cancer Recurrence and Progression in Patients Treated With Bacillus Calmette-Guerin: The CUETO Scoring Model. J Urol 2009;182:2195-203.

10. Cambier S, Sylvester RJ, Collette L, et al. EORTC Nomograms and Risk Groups for Predicting Recurrence, Progression, and Disease-specific and Overall Survival in 
Non-Muscle-invasive Stage Ta-T1 Urothelial Bladder Cancer Patients Treated with 1-3 Years of Maintenance Bacillus Calmette-Guérin. Eur Urol 2016;69:60-9.

11. Chamie K, Ballon-Landa E, Bassett JC, et al. Quality of diagnostic staging in patients with bladder cancer: A process-outcomes link. Cancer 2015;121:379-85.

12. Mariappan P, Zachou A, Grigor KM, et al. Detrusor muscle in the first, apparently complete transurethral resection of bladder tumour specimen is a surrogate marker of resection quality, predicts risk of early recurrence, and is dependent on operator experience. Eur Urol 2010;57:843-9.

13. Richards KA, Smith ND, Steinberg GD. The importance of transurethral resection of bladder tumor in the management of nonmuscle invasive bladder cancer: a systematic review of novel technologies. J Urol 2014;191:1655-64.

14. Babjuk M. Second Resection for Non-Muscle-Invasive Bladder Carcinoma: Current Role and Future Perspectives. Eur Urol 2010;58:191-2.

15. Schraml J, Silva JDC, Babjuk M. Current concept of transurethral resection of bladder cancer: from retransurethral resection of bladder cancer to en-bloc resection. Curr Opin Urol 2018;28:591-7.

16. Jurewicz M, Soloway MS. Approaching the optimal transurethral resection of a bladder tumor. Turk J Urol 2014;40:73-7.

17. Cumberbatch MGK, Foerster B, Catto JWF, et al. Repeat Transurethral Resection in Non-muscle-invasive Bladder Cancer: A Systematic Review. Eur Urol 2018;73:925-33.

18. Naselli A, Hurle R, Paparella S, et al. Role of Restaging Transurethral Resection for T1 Non-muscle invasive Bladder Cancer: A Systematic Review and Meta-analysis. Eur Urol Focus 2018;4:558-67.

19. Herr HW. Role of Re-Resection in Non-Muscle-Invasive Bladder Cancer. ScientificWorldJournal 2011;11:283-8.

20. Herr HW, Donat SM. Quality control in transurethral resection of bladder tumours. BJU Int 2008;102:1242-6.

21. Divrik RT, Şahin AF, Yildirim Ü, et al. Impact of Routine Second Transurethral Resection on the LongTerm Outcome of Patients with Newly Diagnosed pT1 Urothelial Carcinoma with Respect to Recurrence, Progression Rate, and Disease-Specific Survival: A Prospective Randomised Clinical Trial. Eur Urol 2010;58:185-90.

22. Tseng WH, Liao AC, Shen KH, et al. Role of secondlook transurethral resection of bladder tumors for newly diagnosed T1 bladder cancer: Experience at a single center. Urological Science 2018;29:95-9.
23. Liao AH, Tseng WH, Shen KH, et al. Role of secondlook transurethral resection of bladder tumors for newly diagnosed T1 bladder cancer: Experience at a single center. Urological Science 2018. doi: 10.4103/UROS. UROS_17_17.

24. Tseng WH, Liu CL, Huang SK, et al. Therapeutic benefit of second-look transurethral resection of bladder tumors for newly diagnosed T1 bladder cancer: a single-center experience. Int Urol Nephrol 2019;51:1335-42.

25. Gordon PC, Thomas F, Noon AP, et al. Long-term Outcomes from Re-resection for High-risk Non-muscleinvasive Bladder Cancer: A Potential to Rationalize Use. Eur Urol Focus 2019;5:650-7.

26. Krajewski W, Zdrojowy R, Kościelska-Kasprzak K, et al. Does restaging transurethral resection of bladder tumour influence outcomes in patients treated with BCG immunotherapy? 491 cases in 20 years' experience. Wideochir Inne Tech Maloinwazyjne 2019;14:284-96.

27. Woldu SL, Bagrodia A, Lotan Y. Guideline of guidelines: non-muscle-invasive bladder cancer. BJU Int 2017;119:371-80.

28. Gontero P, Sylvester R, Pisano F, et al. The impact of retransurethral resection on clinical outcomes in a large multicentre cohort of patients with T1 high-grade/Grade 3 bladder cancer treated with bacille Calmette-Guérin. BJU Int 2016;118:44-52.

29. Soria F, Marra G, D'Andrea D, et al. The rational and benefits of the second look transurethral resection of the bladder for T1 high grade bladder cancer. Transl Androl Urol 2019;8:46-53.

30. Bos D, Allard CB, Dason S, et al. Impact of resident involvement in endoscopic bladder cancer surgery on pathological outcomes. Scand J Urol 2016;50:234-8.

31. Huang J, Fu J, Zhan H, et al. Analysis of the absence of the detrusor muscle in initial transurethral resected specimens and the presence of residual tumor tissue. Urol Int 2012;89:319-25.

32. Rouprêt M, Yates DR, Varinot J, et al. The presence of detrusor muscle in the pathological specimen after transurethral resection of primary p T1 bladder tumors and its relationship to operator experience. Can J Urol 2012;19:6459-64.

33. Shoshany O, Mano R, Margel D, et al. Presence of detrusor muscle in bladder tumor specimens-predictors and effect on outcome as a measure of resection quality. Urol Oncol. 2014;32:40.e17-22.

34. Aydin A, Shafi AMA, Shamim Khan M, et al. Current Status of Simulation and Training Models in Urological 
Surgery: A Systematic Review. J Urol 2016;196:312-20.

35. Zainfeld D, Daneshmand S. Transurethral Resection of Bladder Tumors: Improving Quality Through New Techniques and Technologies. Curr Urol Rep 2017;18:34.

36. Available online: https://uroweb.org/guideline/nonmuscle-invasive-bladder-cancer/\#5

37. Brausi M. Transurethral Resection of Bladder Cancer: A Simple and Diffusely-Performed Technique but with Controversial Outcomes. Urologia 2013;80:127-9.

38. Burger M, Oosterlinck W, Konety B, et al. ICUD-EAU International Consultation on Bladder Cancer 2012: Nonmuscle-invasive urothelial carcinoma of the bladder. Eur Urol 2013;63:36-44.

39. Nieder AM, Meinbach DS, Kim SS, et al. Transurethral bladder tumor resection: intraoperative and postoperative complications in a residency setting. J Urol 2005;174:2307-9.

40. Panagoda PI, Vasdev N, Gowrie-Mohan S. Avoiding the Obturator Jerk during TURBT. Curr Urol 2018;12:1-5.

41. van der Meijden A, Oosterlinck W, Brausi M, et al. Significance of Bladder Biopsies in Ta,T1 Bladder Tumors: A Report from the EORTC Genito-Urinary Tract Cancer Cooperative Group. Eur Urol 1999;35:267-71.

42. Taguchi I, Gohji K, Hara I, et al. Clinical Evaluation of Random Biopsy of Urinary Bladder in Patients with Superficial Bladder Cancer. Int J Urol 1998;5:30-4.

43. Palou J, Sylvester RJ, Faba OR, et al. Female Gender and Carcinoma In Situ in the Prostatic Urethra Are Prognostic Factors for Recurrence, Progression, and Disease-Specific Mortality in T1G3 Bladder Cancer Patients Treated With Bacillus Calmette-Guérin. Eur Urol 2012;62:118-25.

44. Mungan MU, Canda AE, Tuzel E, et al. Risk factors for mucosal prostatic urethral involvement in superficial transitional cell carcinoma of the bladder. Eur Urol 2005;48:760-3.

45. Beer E. Landmark article May 28, 1910: Removal of neoplasms of the urinary bladder. By Edwin Beer. JAMA 1983;250:1324-5.

46. Puppo P, Bertolotto F, Introini C, et al. Bipolar transurethral resection in saline (TURis): outcome and complication rates after the first 1000 cases. J Endourol 2009;23:1145-9.

47. Wang DS, Bird VG, Leonard VY, et al. Use of Bipolar Energy for Transurethral Resection of Bladder Tumors: Pathologic Considerations. J Endourol 2004;18:578-82.

48. Yang SJ, Song PH, Kim HT. Comparison of Deep Biopsy Tissue Damage from Transurethral Resection of Bladder Tumors between Bipolar and Monopolar Devices. Korean
J Urol 2011;52:379-83.

49. Venkatramani V, Panda A, Manojkumar R, et al. Monopolar Versus Bipolar Transurethral Resection of Bladder Tumors: A Single Center, Parallel Arm, Randomized, Controlled Trial. J Urol 2014;191:1703-7.

50. Gupta NP, Saini AK, Dogra PN, et al. Bipolar energy for transurethral resection of bladder tumours at low-power settings: initial experience. BJU Int 2011;108:553-6.

51. Mashni J, Godoy G, Haarer C, et al. Prospective evaluation of plasma kinetic bipolar resection of bladder cancer: comparison to monopolar resection and pathologic findings. Int Urol Nephrol 2014;46:1699-705.

52. Del Rosso A, Pace G, Masciovecchio S, et al. Plasmakinetic bipolar versus monopolar transurethral resection of nonmuscle invasive bladder cancer: A single center randomized controlled trial. Int J Urol 2013;20:399-403.

53. Xishuang S, Deyong Y, Xiangyu C, et al. Comparing the Safety and Efficiency of Conventional Monopolar, Plasmakinetic, and Holmium Laser Transurethral Resection of Primary Non-muscle Invasive Bladder Cancer. J Endourol 2010;24:69-73.

54. Zhao C, Tang K, Yang H, et al. Bipolar Versus Monopolar Transurethral Resection of Nonmuscle-Invasive Bladder Cancer: A Meta-Analysis. J Endourol 2016;30:5-12.

55. Teoh JY, Chan ES, Yip SY, et al. Comparison of Detrusor Muscle Sampling Rate in Monopolar and Bipolar Transurethral Resection of Bladder Tumor: A Randomized Trial. Ann Surg Oncol 2017;24:1428-34.

56. Garfield SS, Gavaghan MB, Armstrong SO, et al. The cost-effectiveness of blue light cystoscopy in bladder cancer detection: United States projections based on clinical data showing 4.5 years of follow up after a single hexaminolevulinate hydrochloride instillation. Can J Urol 2013;20:6682-9.

57. Witjes JA, Babjuk M, Gontero P, et al. Clinical and cost effectiveness of hexaminolevulinate-guided bluelight cystoscopy: evidence review and updated expert recommendations. Eur Urol 2014;66:863-71.

58. Grossman HB, Stenzl A, Fradet Y, et al. LongTerm Decrease in Bladder Cancer Recurrence with Hexaminolevulinate Enabled Fluorescence Cystoscopy. J Urol 2012;188:58-62.

59. Stenzl A, Burger M, Fradet Y, et al. Hexaminolevulinate Guided Fluorescence Cystoscopy Reduces Recurrence in Patients With Nonmuscle Invasive Bladder Cancer. J Urol 2010;184:1907-13.

60. Burger M, Grossman HB, Droller M, et al. Photodynamic Diagnosis of Non\&\#x2013;muscle-invasive Bladder 
Cancer with Hexaminolevulinate Cystoscopy: A Metaanalysis of Detection and Recurrence Based on Raw Data. Eur Urol 2013;64:846-54.

61. Gakis G, Fahmy O. Systematic Review and Meta-Analysis on the Impact of Hexaminolevulinate- Versus WhiteLight Guided Transurethral Bladder Tumor Resection on Progression in Non-Muscle Invasive Bladder Cancer. Bladder Cancer 2016;2:293-300.

62. Rolevich AI, Zhegalik AG, Mokhort AA, et al. Results of a prospective randomized study assessing the efficacy of fluorescent cystoscopy-assisted transurethral resection and single instillation of doxorubicin in patients with non-muscle-invasive bladder cancer. World J Urol 2017;35:745-52.

63. Mowatt G, N'Dow J, Vale L, et al. Photodynamic diagnosis of bladder cancer compared with white light cystoscopy: Systematic review and meta-analysis. Int J Technol Assess Health Care 2011;27:3-10.

64. Daneshmand S, Bazargani ST, Bivalacqua TJ, et al. Blue light cystoscopy for the diagnosis of bladder cancer: Results from the US prospective multicenter registry. Urol Oncol 2018;36:361.e1-e6.

65. Daneshmand S, Patel S, Lotan Y, et al. Efficacy and Safety of Blue Light Flexible Cystoscopy with Hexaminolevulinate in the Surveillance of Bladder Cancer: A Phase III, Comparative, Multicenter Study. J Urol 2018;199:1158-65.

66. Inoue K, Fukuhara H, Shimamoto T, et al. Comparison between intravesical and oral administration of 5 -aminolevulinic acid in the clinical benefit of photodynamic diagnosis for nonmuscle invasive bladder cancer. Cancer 2012;118:1062-74.

67. Chou R, Selph S, Buckley DI, et al. Comparative Effectiveness of Fluorescent Versus White Light Cystoscopy for Initial Diagnosis or Surveillance of Bladder Cancer on Clinical Outcomes: Systematic Review and Meta-Analysis. J Urol 2017;197:548-58.

68. Li K, Xu Y, Tan M, et al. A retrospective comparison of thulium laser en bloc resection of bladder tumor and plasmakinetic transurethral resection of bladder tumor in primary non-muscle invasive bladder cancer. Lasers Med Sci 2019;34:85-92.

69. Zheng C, Lv Y, Zhong Q, et al. Narrow band imaging diagnosis of bladder cancer: systematic review and metaanalysis. BJU Int 2012;110:E680-7.

70. Li K, Lin T, Fan X, et al. Diagnosis of narrow-band imaging in non-muscle-invasive bladder cancer: A systematic review and meta-analysis. Int J Urol
2013;20:602-9.

71. Naito S, Algaba F, Babjuk M, et al. The Clinical Research Office of the Endourological Society (CROES) Multicentre Randomised Trial of Narrow Band ImagingAssisted Transurethral Resection of Bladder Tumour (TURBT) Versus Conventional White Light ImagingAssisted TURBT in Primary Non-Muscle-invasive Bladder Cancer Patients: Trial Protocol and 1-year Results. Eur Urol 2016;70:506-15.

72. Lee JY, Cho KS, Kang DH, et al. A network meta-analysis of therapeutic outcomes after new image technologyassisted transurethral resection for non-muscle invasive bladder cancer: 5-aminolaevulinic acid fluorescence vs hexylaminolevulinate fluorescence vs narrow band imaging. BMC Cancer 2015;15:566.

73. Drejer D, Béji S, Oezeke R, et al. Comparison of White Light, Photodynamic Diagnosis, and Narrow-band Imaging in Detection of Carcinoma In Situ or Flat Dysplasia at Transurethral Resection of the Bladder: the DaBlaCa-8 Study. Urology 2017;102:138-42.

74. Kramer MW, Altieri V, Hurle R, et al. Current Evidence of Transurethral En-bloc Resection of Nonmuscle Invasive Bladder Cancer. Eur Urol Focus 2017;3:567-76.

75. Kawada T. A New Technique for Transurethral Resection of Bladder Tumors: Rotational Tumor Resection Using a New Arched Electrode. J Urol 1997;157:2225-6.

76. Fritsche HM, Otto W, Eder F, et al. Water-Jet-Aided Transurethral Dissection of Urothelial Carcinoma: A Prospective Clinical Study. J Endourol 2011;25:1599-603.

77. Bach T, Muschter R, Herrmann TRW, et al. Technical solutions to improve the management of non-muscleinvasive transitional cell carcinoma: summary of a European Association of Urology Section for UroTechnology (ESUT) and Section for Uro-Oncology (ESOU) expert meeting and current and future perspectives. BJU Int 2015;115:14-23.

78. Sureka SK, Agarwal V, Agnihotri S, et al. Is en-bloc transurethral resection of bladder tumor for non-muscle invasive bladder carcinoma better than conventional technique in terms of recurrence and progression? A prospective study. Indian J Urol 2014;30:144-9.

79. Hurle R, Lazzeri M, Colombo P, et al. "En Bloc" Resection of Nonmuscle Invasive Bladder Cancer: A Prospective Single-center Study. Urology 2016;90:126-30.

80. Zhang KY, Xing JC, Li W, et al. A novel transurethral resection technique for superficial bladder tumor: retrograde en bloc resection. World J Surg Oncol 2017;15:125. 
81. Zhang J, Wang L, Mao S, et al. Transurethral en bloc resection with bipolar button electrode for non-muscle invasive bladder cancer. Int Urol Nephrol 2018;50:619-23.

82. Nagele U. New techniques for bladder tumor resection: are they better than conventional TURBT? Eur Urol Supplements 2018;17:56-9.

83. Gakis G, Karl A, Bertz S, et al. Transurethral en-bloc hydrodissection for non-muscle invasive bladder cancer: Results of a randomized controlled trial. Eur Urol Supplements 2017;16:e1143-4.

84. Wu YP, Lin TT, Chen SH, et al. Comparison of the efficacy and feasibility of en bloc transurethral resection of bladder tumor versus conventional transurethral resection of bladder tumor. Medicine (Baltimore) 2016;95:e5372.

85. Yang H, Wang N, Han S, et al. Comparison of the efficacy and feasibility of laser enucleation of bladder tumor versus transurethral resection of bladder tumor: a meta-analysis. Lasers Med Sci 2017;32:2005-12.

86. Naselli A, Puppo P. En Bloc Transurethral Resection of Bladder Tumors: A New Standard? J Endourol 2017;31:S20-4.

87. Kramer MW, Rassweiler JJ, Klein J, et al. En bloc resection of urothelium carcinoma of the bladder (EBRUC): a European multicenter study to compare safety, efficacy, and outcome of laser and electrical en bloc transurethral resection of bladder tumor. World J Urol 2015;33:1937-43.

88. Migliari R, Buffardi A, Ghabin H. Thulium Laser Endoscopic En Bloc Enucleation of Nonmuscle-Invasive Bladder Cancer. J Endourol 2015;29:1258-62.

89. Chen X, Liao J, Chen L, et al. En bloc transurethral resection with 2-micron continuous-wave laser for primary non-muscle-invasive bladder cancer: a randomized controlled trial. World J Urol 2015;33:989-95.

90. He D, Fan J, Wu K, et al. Novel Green-Light KTP Laser En Bloc Enucleation for Nonmuscle-Invasive Bladder Cancer: Technique and Initial Clinical Experience. J Endourol 2014;28:975-9.

91. Muto G, Collura D, Giacobbe A, et al. Thulium:yttriumaluminum-garnet Laser for En Bloc Resection of Bladder Cancer: Clinical and Histopathologic Advantages. Urology 2014;83:851-5.

92. Upadhyay R, Kapoor R, Srivastava A, et al. Does Enbloc transurethral resection of bladder tumor give a better yield in terms of presence of detrusor muscle in the biopsy specimen? Indian J Urol 2012;28:275-9.

93. Maurice MJ, Vricella GJ, MacLennan G, et al. Endoscopic Snare Resection of Bladder Tumors: Evaluation of an Alternative Technique for Bladder Tumor Resection. J
Endourol 2012;26:614-7.

94. Nagele U, Kugler M, Nicklas A, et al. Waterjet hydrodissection: first experiences and short-term outcomes of a novel approach to bladder tumor resection. World J Urol 2011;29:423-7.

95. Ukai R, Hashimoto K, Iwasa T, et al. Transurethral resection in one piece (TURBO) is an accurate tool for pathological staging of bladder tumor. Int J Urol 2010;17:708-14.

96. Lodde M, Lusuardi L, Palermo S, et al. En bloc transurethral resection of bladder tumors: use and limits. Urology 2003;62:1089-91.

97. Saito S. Transurethral en bloc resection of bladder tumors. J Urol 2001;166:2148-50.

98. Zhang XR, Feng C, Zhu WD, et al. Two Micrometer Continuous-Wave Thulium Laser Treating Primary Non-Muscle-Invasive Bladder Cancer: Is It Feasible? A Randomized Prospective Study. Photomed Laser Surg 2015;33:517-23.

99. Zhu Y, Jiang X, Zhang J, et al. Safety and Efficacy of Holmium Laser Resection for Primary NonmuscleInvasive Bladder Cancer Versus Transurethral Electroresection: Single-Center Experience. Urology 2008;72:608-12.

100. Liu H, Wu J, Xue S, et al. Comparison of the safety and efficacy of conventional monopolar and 2-micron laser transurethral resection in the management of multiple nonmuscle-invasive bladder cancer. J Int Med Res 2013;41:984-92.

101. Chen J, Zhao Y, Wang S, et al. Green-light laser en bloc resection for primary non-muscle-invasive bladder tumor versus transurethral electroresection: A prospective, nonrandomized two-center trial with 36-month follow-up. Lasers Surg Med 2016;48:859-65.

102.Hermann GG, Mogensen K, Rosthoj S. Outpatient diode laser treatment of intermediate-risk non-invasive bladder tumors without sedation: efficacy, safety and economic analysis. Scand J Urol 2018;52:194-8.

103. Hayashida Y, Miyata Y, Matsuo T, et al. A pilot study to assess the safety and usefulness of combined transurethral endoscopic mucosal resection and en-bloc resection for non-muscle invasive bladder cancer. BMC Urol 2019;24;19:56.

Cite this article as: Kim LHC, Patel MI. Transurethral resection of bladder tumour (TURBT). Transl Androl Urol 2020;9(6):3056-3072. doi: 10.21037/tau.2019.09.38 